\title{
Predicting fluid responsiveness in non-intubated COVID-19 patients
}

\author{
Frederic Michard ${ }^{*}$ (i)
}

I read with great interest the article by Caplan et al. [1] about the prediction of fluid responsiveness in spontaneously breathing patients. They nicely showed that when patients perform a standardized respiratory maneuver and the inferior vena cava (IVC) diameter measurements are done precisely $4 \mathrm{~cm}$ from the cavo-atrial junction, the magnitude of the IVC respiratory variation becomes highly predictive of fluid responsiveness.

The current COVID-19 pandemic is responsible for a surge of patients hospitalized for hypoxemic pneumonia. Most of them are spontaneously breathing and stay on hospital wards where they receive oxygen. In this context of acute respiratory failure (ARF), excessive fluid administration may increase pulmonary leak, worsen arterial hypoxemia and precipitate tracheal intubation. On the other hand, insufficient fluid administration may promote the development of acute kidney injury and hemodynamic instability. Therefore, in severe COVID-19 cases, predicting fluid responsiveness is recommended by international and WHO guidelines.

At first sight, the method proposed by Caplan et al. [1] is appealing because it is non-invasive and associated with a high predictive value. However, in practice, it may be difficult to implement for several reasons. First, patients with ARF may have difficulties to cooperate and standardize the way they breath. Second, ARF patients are usually sitting in their bed, with significant respiratory abdomen movements, which does not facilitate the assessment of IVC variations from a sub-costal

See relatedresearch by Morgan Caplan, Arthur Durand, Perrine Bortolotti et al.https://annalsofintensivecare.springeropen.com/articles/10.1186/s1361 3-020-00786-1

*Correspondence: frederic.michard@bluewin.ch

MiCo, Denens, Switzerland view - not mentioning the fact that obesity is common in COVID-19 patients. Would Caplan et al. [1] recommend their method in this specific clinical context? Third, because echocardiography is increasingly used by noncardiologists, would Caplan et al. [1] agree with the fact that the level of precision in IVC diameter measurements requested by their method (exactly $4 \mathrm{~cm}$ from the cavoatrial junction) may be a challenge for some operators?

One should also consider that significant respiratory efforts may induce dramatic changes in intrathoracic pressure and venous return. As a result, in spontaneously breathing patients with ARF, respiratory variations in IVC diameter, or in blood pressure (pulsus paradoxus), or in the pulse oximetry waveform, may depend more on the magnitude of respiratory efforts than on the volume status [2]. I noticed that Caplan et al. [1] excluded patients with active expiration from their evaluation. Do they agree that their method may also have limitations in patients making significant inspiratory efforts?

In patients without any clinical and biological signs of shock or acute kidney injury, a fluid restriction strategy is recommended to limit the development of pulmonary edema and prevent ICU admission. In contrast, in patients with hemodynamic instability or biological signs of tissue hypoperfusion, it may be wise to perform a passive leg raising (PLR) maneuver to identify patients who may benefit from receiving fluid (Fig. 1). It is now well established that the lack of increase in blood pressure during a PLR maneuver cannot exclude a significant improvement in blood flow and oxygen delivery. It is therefore recommended to continuously monitor left ventricular stroke volume in order to assess the hemodynamic response to PLR [3].

In patients who have an arterial catheter in place, a pulse contour technique can be proposed to track 


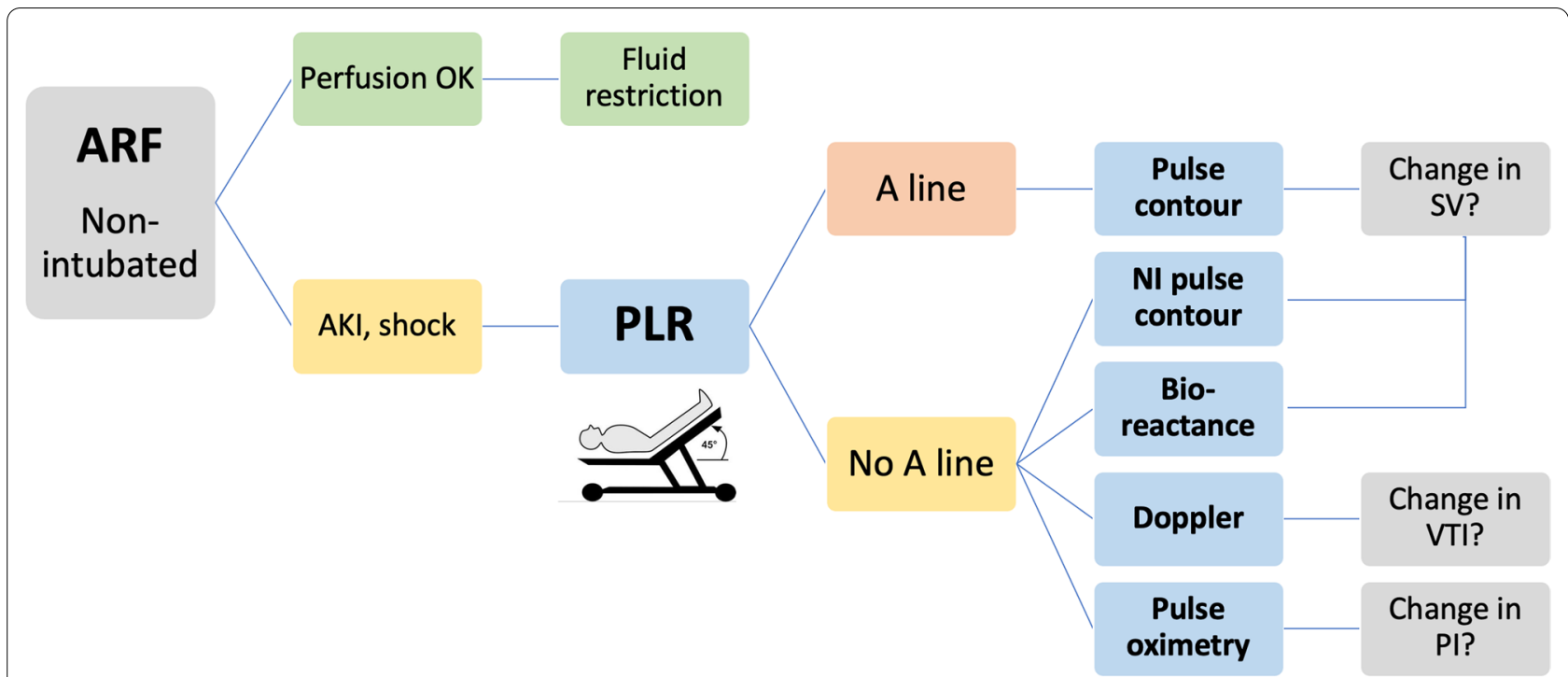

Fig. 1 How to predict fluid responsiveness in non-intubated patients with acute respiratory failure (ARF). PLR, passive leg raising maneuver; A line, arterial line; NI, non-invasive; SV, stroke volume; VTI, velocity time integral; PI, perfusion index

changes in stroke volume during the PLR maneuver. In patients who do not have an arterial catheter (most spontaneously breathing patients), there are today basically three options (Fig. 1). The first one is to use a noninvasive cardiac output monitoring system, either a pulse contour technique or a bioreactance method. Both have been shown to be useful to track changes in stroke volume during a PLR maneuver. The second option is to track changes in blood velocity time integral (VTI) using echo-Doppler. It can be done either with a Point Of Care UltraSound (POCUS) device from a thoracic view or with a Doppler probe in the supra-sternal area. In the future, one may also use a wireless Doppler patch positioned on the carotid artery [4].

When none of the above sophisticated systems is available (which is today the case in most hospital wards), one may consider using a pulse oximeter (Fig. 1). Indeed, in addition to $\mathrm{SpO}_{2}$ and pulse rate numbers, pulse oximeters display the peripheral perfusion index (PI). The two main determinants of PI are vascular tone and stroke volume. A brief mechanical maneuver like PLR is unlikely to induce significant changes in vascular tone. As a result, it has recently been shown that tracking changes in PI may replace the direct assessment of stroke volume during a PLR maneuver [5].

In summary, although the standardized method described by Caplan et al. [1] may have value to predict fluid responsiveness in calm, cooperative and nonobese patients, it may be difficult to use in ARF patients in real life conditions. With the current surge of hypoxemic COVID-19 patients, clinicians need more than ever simple solutions to rationalize fluid therapy and improve quality of care.

\section{Acknowledgments}

Not applicable.

\section{Authors' contributions}

FM wrote the manuscript. The author read and approved the final manuscript.

Funding

Not applicable.

Availability of data and materials

Not applicable.

Ethics approval and consent to participate

Not applicable.

\section{Consent for publication}

Not applicable.

\section{Competing interests}

FM is the managing director of MiCo (michardconsulting.com), a Swiss consulting and research firm. MiCo does not sell any medical products and FM does not own shares from any MedTech company.

Received: 12 January 2021 Accepted: 18 January 2021

Published online: 27 January 2021

\section{References}

1. Caplan M, Durand A, Bortolotti P, et al. Measurement site of inferior vena cava diameter affects the accuracy with which fluid responsiveness can be predicted in spontaneously breathing patients: a post hoc analysis of two prospective cohorts. Ann Intensive Care. 2020;10(1):168

2. Michard F, Shelley K. Should we monitor pulse paradoxus via pulse oximetry in patients with COVID-19 and acute respiratory failure? Am J Respir Crit Care Med. 2020;202(5):770-1. 
3. Monnet $X$, Teboul JL. Passive leg raising: five rules, not a drop of fluid. Crit Care. 2015;19(1):18.

4. Kenny JS, Barjaktarevic I, Eibl AM, et al. A carotid Doppler patch accurately tracks stroke volume changes during a preload-modifying maneuver in healthy volunteers. Crit Care Explor. 2020;2(1):e0072.

5. Beurton A, Teboul JL, Gavelli F, et al. The effects of passive leg raising may be detected by the plethysmographic oxygen saturation signal in critically ill patients. Crit Care. 2019;23(1):19.

\section{Publisher's Note}

Springer Nature remains neutral with regard to jurisdictional claims in published maps and institutional affiliations.

\section{Submit your manuscript to a SpringerOpen ${ }^{\circ}$ journal and benefit from:}

- Convenient online submission

- Rigorous peer review

- Open access: articles freely available online

- High visibility within the field

- Retaining the copyright to your article

Submit your next manuscript at $\boldsymbol{\sim}$ springeropen.com 